\title{
The Relation between circulating endothelial microparticles and carotid intima media thickness in Vitamin B12 deficient adolescents
}

\author{
Mehmet Akif Dündar1*, Yasemin Altuner Torun², Feyza Çetin ${ }^{3}$, Serkan Fazlı Çelik ${ }^{4}$, Çiğdem Karakükçü ${ }^{5}$ and Emine Kaygı Tartıcı ${ }^{1}$ \\ ${ }^{1}$ Medical Health University Kayseri Training and Research Hospital, Department of Pediatrics, Kayseri, Turkey \\ ${ }^{2}$ Medical Health University Kayseri Training and Research Hospital, Department of Pediatrics, Division of Pediatric Hematology, Kayseri, Turkey \\ ${ }^{3}$ Medical Health University Kayseri Training and Research Hospital, Department of Microbiology, Kayseri, Turkey \\ ${ }^{4}$ Medical Health University Kayseri Training and Research Hospital, Department of Pediatrics, Division of Pediatric Cardiology, Kayseri, Turkey \\ ${ }^{5}$ Medical Health University Kayseri Training and Research Hospital, Department of Biochemistry, Kayseri, Turkey
}

\begin{abstract}
Background and aims: Endothelial microparticles (EMPs) thought to play a key role related to endothelial dysfunction. Carotid intima-media thickness (cIMT) is an early indicator of atherosclerosis. The aim of our study is to investigate the levels of cIMT, EMP which are indicative of susceptibility to atherosclerosis and endothelial dysfunction in adolescents with deficiency of vitamin B12.

Methods: Adolescent with age ranging between 11-17 years age eighty-eight patients were enrolled 50 patients of vitamin B12 deficiency (B12<130 pg/ml) and 38 healthy controls $(\mathrm{B} 12>200 \mathrm{pg} / \mathrm{ml})$ were included in the study. In all cases number of EMPs (CD144+EMP, CD146+EMP, CD105+EMP) were measured in the flow cytometry. The cIMT were assessed using high-resolution echocardiography.

Results: Both plasma levels of CD144+EMP, CD146+EMP and cIMT (mm) were significantly higher in vitamin B12 deficiency group than control group. Correlation analysis showed vitamin B12 was significant negative correlation with homocysteine, CD144+EMP, CD146+EMP and cIMT (p<0.05).

Conclusions: Vitamin B12 deficient adolescents had higher plasma EMP levels and cIMT values than the controls, reflecting the presence of subclinical atherosclerosis. Vitamin B12 deficiency may also contribute endothelial dysfunction and atherosclerosis. CD144+ and CD146+ EMP may be used as markers for predicting atherosclerosis and endothelial dysfunction in adolescents with vitamin B12 deficiency.
\end{abstract}

\section{Introduction}

Vitamin B12 deficiency leads to a decrease in methylenetetrahydro-folate reductase (MTHFR) activity that reduces synthesis of methionine and occurs accumulation of homocysteine [1]. Elevated levels of plasma homocysteine are an independent risk factor for affecting atherosclerosis and coronary artery disease $[2,3]$.

Oxidative stress refers to the imbalance between oxidant products and antioxidant defense mechanisms. Reactive oxygen radicals stimulate proliferation in vascular smooth muscle cells and release of proinflammatory cytokines by reducing NO levels [4,5]. Microparticles are small $(0.2-2 \mu \mathrm{m})$ vesicles released by cellular stress and activation by many eukaryotic cells such as platelets, leukocytes and endothelial cells [6].

In recent years, these microparticles have been shown to be useful markers in pathological conditions such as vascular inflammation, coagulation, and tumoral diseases [7]. Circulating endothelial microparticles (EMPs) are considered to reflect the endothelial injury and vascular inflammation. The number of EMP circulating in the peripheral blood are associated with atherosclerosis and several cardiovascular risk factors [8].

Carotid intima media thickness (cIMT) is a noninvasive, simple method of predicting future vascular risk factors such as atherosclerosis, coronary artery disease [9]. Determination of the cIMT by echocardiography is a simple, inexpensive, noninvasive and correlated technique [10].

In this study, we aimed to investigate the predisposition of endothelial dysfunction and atherosclerosis, to examine the relationship between EMP and cIMT in adolescents with vitamin B12 deficiency.

\section{Material and methods}

This case-control study included 50 vitamin B12 deficient adolescents and 38 healthy subjects aged between 11 and 18 years. The diagnosis of severe vitamin B12 deficiency was based on serum vitamin B12 level of $<130 \mathrm{pg} / \mathrm{mL}$ and a normal level of folic acid $[11,12]$. The control group vitamin B12 level greater than $200 \mathrm{pg} / \mathrm{ml}$. The local institutional ethics committee approved the study protocol. Informed consent was obtained from all subjects.

${ }^{*}$ Correspondence to: Mehmet Akif Dündar, Medical Health University Kayseri Training and Research Hospital, Department of Pediatrics, Kayseri, Turkey; E-mail: doktordundar1984@gmail.com

Key words: atherosclerosis, endothelial microparticles, vitamin B12 deficiency

Received: July 05, 2018; Accepted: July 25, 2018; Published: August 02, 2018 
Blood samples were taken after 8 hours fasting from all cases. $\mathrm{CBC}$, vitamine $\mathrm{B} 12$, folic acid, ferritin, homocysteine, triglyceride, total cholesterol, HDL-cholesterol, LDL-cholesterol, LDH, iron and iron binding capacity were immediately studied in the blood samples taken from the blood samples. The blood samples were drawn in 0.129 $\mathrm{mol} / \mathrm{l}$ sodium citrate tubes. Platelet free plasma (PFP) was prepared as previously described [13]. The samples were centrifuged for $15 \mathrm{~min}$ at $1500 \mathrm{~g}$ and plasma was then harvested and centrifuged for $2 \mathrm{~min}$ at $13000 \mathrm{~g}$. PFP was stored at $-80^{\circ} \mathrm{C}$ until used.

A special program, Vivid 7 / Vivid i IMT Package, was used in the echocardiography device to measure the cIMT. This measurement procedure is not operator-dependent and automatically measures the mean cIMT after the device has detected the carotid artery.

CD144+EMP is endothelial specific and CD146+ EMP is released from endothelial and activated T lymphocytes. EMPs were conjugated with 3 different antibodies and isotypes and analyzed at rates below 10000 events / second in the Flow cytometry device and 1.000.000 events were collected in the microparticle door (Figure 1).

\section{Statistical analysis}

All tests were performed using SPSS for Windows 22.0 and Sigma Stat 3.1. First, the distributions of all parameters were determined by using the Shapiro-Wilk test. The parameters with normal distribution were expressed as mean $\pm \mathrm{SD}$, and the parameters with abnormal distribution were expressed as median (25th percentile-75th percentile). Comparisons of means were performed with Student's t-test. Comparisons of medians were performed with the MannWhitney U-test. Correlations were calculated with the Pearson product moment or Spearman rank order, as determined by the normalcy of data distribution. Two multiple regression analyse and binary logistic regression were performed. A P-value of $<0.05$ was accepted as statistically significant.

\section{Results}

The epidemiological and biochemical characteristics, EMPs results, and vascular measurements of the study groups are shown in Table 1. There were no statistical differences between the both groups regarding age and gender distribution. Vitamin B12 levels were statistically lower in the patient's group than in the control group $(\mathrm{p}<0.05$, Table 1$)$. Homocysteine were statistically higher in the patient's group than in the control group $(\mathrm{p}<0.05$, Table 1$)$. The median number of CD 144+ EMP and CD146 + EMP were significantly higher in children with vitamin B12 deficiency children than healthy controls. There was no statistically significant difference between patients and control groups in terms of CD105 + EMP. cIMT was statistically higher in the patient's group than in the control group $(\mathrm{p}<0.05$, Table 1$)$.

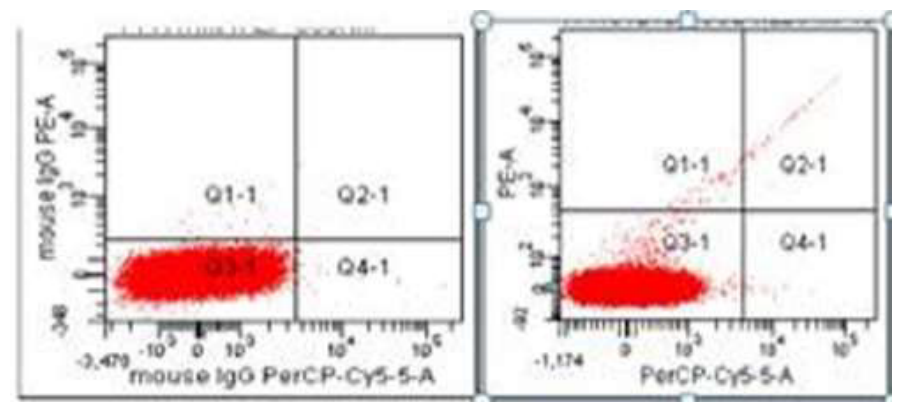

Figure 1. Flow cytometry EMP measurement: The plasma of each patient was evaluated with isotype and the negative states were accurately identified
Table 1. Demographic and biochemical characteristics, EMPs results, and vascular measurements of study groups. Data are given as mean \pm SD and as median (Q1-Q3)

\begin{tabular}{|l|l|l|l|}
\hline Parameters & $\begin{array}{l}\text { Vitamin B12 } \\
\text { Deficiency } \\
\text { (n=50) }\end{array}$ & $\begin{array}{l}\text { Control } \\
\text { (n=38) }\end{array}$ & $\mathbf{P}$ \\
\hline Gender, male/female & $\mathbf{2 0 / 3 0}$ & $15 / 23$ & $>\mathbf{0 . 0 5}$ \\
\hline Age, years & $14.4 \pm 1.72$ & $13.4 \pm 1.86$ & $>\mathbf{0 . 0 5}$ \\
\hline Vitamin B12 (pg/ml) & $100(85-115)$ & $238(210-277)$ & $<\mathbf{0 . 0 5}$ \\
\hline Homocysteine $(\mathbf{m m o l} / \mathbf{L})$ & $21 \pm 8.06$ & $10 \pm 2.54$ & $<\mathbf{0 . 0 5}$ \\
\hline CD144+EMP $(\mathbf{n} /<\mathbf{0 . 0 5} \mathbf{L})$ & $16.26(8.91-34.72)$ & $9.51(5.01-19.34)$ & $<\mathbf{0 . 0 5}$ \\
\hline CD146+EMP $(\mathbf{n} / \boldsymbol{\mu L})$ & $48.11(22.12-137.03)$ & $21.44(10.43-33.6)$ & $<\mathbf{0 . 0 0 1}$ \\
\hline CD105+EMP $(\mathbf{n} / \boldsymbol{\mu L})$ & $107.6(50.9-314,4)$ & $83.61(50.4-145.2)$ & $>0.05$ \\
\hline cIMT $(\mathbf{m m})$ & $0.87(0.69-1.21)$ & $0.68(0.52-0.80)$ & $<\mathbf{0 . 0 0 1}$ \\
\hline
\end{tabular}

The study showed an inverse relation between vitamin $\mathrm{B} 12$ value and homocysteine, cIMT, CD144+EMP and CD146+EMP numbers ( $\mathrm{p}$ $<0.001$, Table 2).

The binary logistic regression analysis and the backward elimination method showed that cIMT,and homocystein have a linear relationship with vitamine B12 deficiency $(\mathrm{p}<0.05$, Table 3$)$.

There was positive relation between cIMT and homocysteine, CD144+EMP and CD146+ EMP $(\mathrm{p}<0.05)$. In addition, an inverse relation between cIMT and vitamin B12 ( $\mathrm{p}<0.05$, Table 2$)$. The multiple regression analysis between cIMT and homocystein and $\mathrm{LDH}$, vitamin B12 showed a relationship between cIMT and homocystein (Table 4).

\section{Discussion}

The main findings of our preliminary study can be summarized as follows: 1) increased EMP and homocystein levels were observed in adolescents with vitamin B12 deficiency, 2) In addition, vitamin B12 deficient adolescents have increased cIMT, 3) the levels of circulating CD 105+ EMP in patients with vitamin B12 deficiency and those without were similar.

Vitamin B12 deficiency and elevated homocysteine affects endothelial dysfunction, vascular wall changes and leads to cardiovascular and atherogenic risk (3). The most important factor determining homocysteine as vitamin B12 and vitamin B12 deficiency had adverse effects on cardiovascular disease.

Vitamin B12 supplementation should be used to reduce the risk of cardiovascular disease [14]. According to the study by Harker, et al. [15], homocysteine is a reactive amino acid and initiates premature atherosclerosis and endothelial dysfunction as early as 4 years. Because of their close relationship homocysteine levels can be an indirect indication of a vitamin B12 deficiency. Elevated homocysteine levels, also known as homocysteinemia, is often an indication of a B12 deficiency [16]. In the present study, we observed increased homocystein in children with vitamin B12 deficiency. Therefore, an increased homocystein level may reflect endothelial dysfunction in vitamin B12 deficiency.

In many experimental and cross-sectional studies, cIMT has been reported as an early indicator of atherosclerosis [17]. Pignoli et al. [18] reported that changes in the cIMT may be helpful in predicting cardiovascular events. Libetta et al. [19] showed patients with high homocysteine levels undergoing renal transplantation found that cIMT was significantly higher than normal population. We found that vitamin B12 deficient adolescents have significantly higher cIMT (Table 1). If the increase in cIMT is considered a marker of atherosclerosis, it can be assumed that atherosclerotic changes have begun with a decrease in vitamin B12. In this study, we performed binary logistic regression analyses by designating vitamin B12 deficiency as a dependent variable and EMP level, cIMT and homocystein as the independent variables 
Table 2. Correlation between vitamin B12, cIMT and other variables

\begin{tabular}{|c|c|c|c|c|}
\hline \multirow{2}{*}{ Variables } & \multicolumn{2}{|c|}{ Vitamin B12 } & \multicolumn{2}{|c|}{ cIMT } \\
\hline & $\mathbf{r}$ & $\mathbf{p}$ & $\mathbf{r}$ & $\mathbf{p}$ \\
\hline Homocysteine (mmol / L) & -0.58 & $<0.001$ & 0.50 & $<0.001$ \\
\hline CD144+EMP (n/ $\mu \mathrm{L})$ & -0.24 & $<0.05$ & 0.23 & $<0.05$ \\
\hline $\operatorname{CD146+EMP(n/\mu L)}$ & -0.31 & $<0.05$ & 0.29 & $<0.05$ \\
\hline $\operatorname{CD105}+\operatorname{EMP}(n / \mu L)$ & -0.06 & $>0.05$ & 0.02 & $>0.05$ \\
\hline Folate (ng/ml) & 0.24 & $<0.05$ & 0.38 & $>0.05$ \\
\hline HDL (mg/dl) & 0.29 & $<0.05$ & 0.21 & $>0.05$ \\
\hline Vitamine B12 (pg/mL) & - & - & -0.27 & $<0.05$ \\
\hline
\end{tabular}

Table 3. Binary logistic regression analysis between vitamin B12 deficiency and other variables (OR: Odd's Ratio, CI: Confidence Interval)

\begin{tabular}{|c|c|c|c|c|}
\hline \multicolumn{5}{|c|}{ Dependent variable vitamin B12 deficiency } \\
\hline \multirow{2}{*}{ (Independent variables) } & \multicolumn{2}{|c|}{ Univarite Logistic } & \multicolumn{2}{|c|}{ Multiple Logistic } \\
\hline & OR $(\% 95 \mathrm{CI})$ & $\mathbf{P}$ & OR $(\% 95 \mathrm{CI})$ & $\mathbf{P}$ \\
\hline CD144+EMP & $1.00(1.00-1.01)$ & 0.57 & - & - \\
\hline CD146+EMP & $1.02(1.01-1.03)$ & 0.008 & - & - \\
\hline CD105+ EMP & $1.01(1.0-1.04)$ & 0.92 & - & - \\
\hline cìMT & $31.79(4.53-222)$ & $<0.001$ & $16.17(1.00-266)$ & $<0.05$ \\
\hline Homocystein & $1.53(1.26-1.87)$ & $<0.001$ & $1.59(1.4-2.02)$ & $<0.001$ \\
\hline TRİGLİSERİT & $1.008(1.00-1.016)$ & 0.50 & $1.01(1.0-1.02)$ & $<0.05$ \\
\hline HDL & $0.92(0.88-0.97)$ & 0.002 & $0.9(0.82-0.99)$ & $<0.05$ \\
\hline LDH & $0.98(0.97-1.00)$ & 0.05 & - & - \\
\hline
\end{tabular}

Table 4. Multiple regression analysis between cIMT and other parameters

\begin{tabular}{|c|c|c|c|c|c|c|c|}
\hline \multirow{3}{*}{ Parameters } & \multicolumn{7}{|c|}{$\operatorname{cIMT}(\mathrm{n}=\mathbf{8 8})$} \\
\hline & \multicolumn{2}{|c|}{ Coefficient } & \multirow[b]{2}{*}{$\mathbf{T}$} & \multirow[b]{2}{*}{$\mathbf{P}$} & \multirow[b]{2}{*}{$\mathbf{F}$} & \multirow[b]{2}{*}{ Model (P) } & \multirow{2}{*}{$\mathbf{R}^{2}$} \\
\hline & B & S. error & & & & & \\
\hline Constant & 1.11 & 0.3 & 4.82 & $<0.001$ & \multirow{6}{*}{4.45} & \multirow{6}{*}{0.003} & \multirow{6}{*}{0.17} \\
\hline Homocysteine & 0.01 & 0.005 & 2.39 & 0.01 & & & \\
\hline LDH & -0.002 & -0.001 & -1.62 & 0.10 & & & \\
\hline B12 & -0.001 & 0.001 & -1.5 & 0.13 & & & \\
\hline CD 144+ EMP & -0.27 & 0.32 & -0.83 & 0.40 & & & \\
\hline CD 146+ EMP & $-0,32$ & 0.25 & $-0,78$ & 0.38 & & & \\
\hline
\end{tabular}

(Tables 3). After the backward stepwise elimination, we detected that an increased cIMT, homocysteine has a linear relationship with vitamin B12 deficiency.

Studies and standardizations on EMPs are important in terms of its utility in vascular health, atherosclerosis and endothelial dysfunction and a new noninvasive test [13]. Bruyndonckx, et al. [20] measured the circulating level of CD42b + and CD144+EMP in obese children. They found that the circulating levels of these markers in obese children were significantly higher than those in the healthy controls. In another study, Suhara, et al. [21] hyperhomocysteinemia increase EMPs by increasing apoptosis in Fas ligand-mediated endothelial cells. Additionally, Sekula, et al. [22] found that CD31 and annexinV EMPs were significantly higher in patients MTHFR polymorphism with homocysteine increase.

There are no current data on EMPs in adolescents with vitamin B12 deficiency. In our study, it was found that the CD144 + EMP and CD146 + EMP number were higher in vitamin B12 deficient adolescents than in the control group. There was no difference in CD105 + EMPs between groups (Table 1). Therefore, we think that high circulating CD144 + EMP and CD146 + EMP levels may be correlated with endothelial dysfunction and atherosclerosis. EMPs are long term centrifuges, marking, placement in tubes and scaling in flow cytometry devices are difficult and require expertise. Because the dimensions of the EMPs are very small and flow cytometry is the best method for detecting EMPs. It would be more useful to have new methods that can detect EMPs precisely and more easily.
'Our study has some limitations. First, it will be more useful to reevaluate patients after vitamin B12 treatment. Second methylmalonic acid is used as a sensitive test for vitamin B12 deficiency, was not measured in this study. Finally, multicentric studies should be performed to use EMP as a determinant in clinical practice.

\section{Conclusion}

In conclusion, our results show that vitamin B12 deficient adolescents have increased circulating CD144 + EMP, CD146 + EMP showing that endothelial dysfunction. In addition, cIMT increases in adolescents with vitamin B12 deficiency. EMP may be used as markers for predicting atherosclerosis and endothelial dysfunction. Vitamine B12 deficiency may also contribute endothelial dysfunction and atherosclerosis.

\section{Ethics committee approval}

Ethics committee approval was received for this study from Erciyes

University ethics committee at 19/02/2016 with the record number of 115 .

\section{Informed consent}

Written informed consent was obtained from patients who participated in this study. 


\section{Conflict of Interest}

No conflict of interest was declared by the authors.

\section{Financial Disclosure}

The authors declared that this study has received no financial support.

\section{References}

1. Lorenz MW, Markus HS, Bots ML, Rosvall M, Sitzer M (2007) Prediction of clinica cardiovascular events with carotid intima-media thickness a systematic review and meta-analysis. Circulation 115: 459-467. [Crossref]

2. Genest JJ Jr, McNamara JR, Salem DN, Wilson PW, Schaefer EJ, et al. (1990) Plasma homocyst(e)ine levels in men with premature coronary artery disease. $J$ Am Coll Cardiol 16: 1114-1119. [Crossref]

3. Virdis A, Ghiadoni L, Salvetti G, Versari D, Taddei S, et al. (2001) Hyperhomocysteinemia: Is a novel risk factor in hypertension? J Nephrol 15: 414-421.

4. Zoccali C, Bode-Boger S, Mallamaci F, Benedetto F, Tripepi G, et al. (2001) Plasma concentration of asymmetrical dimethylarginine and mortality in patients with endstage renal disease: a prospective study. Lancet 358: 2113-2117. [Crossref]

5. Schiro A, Wilkinson FL, Weston R, Smyth JV, Serracino-Inglott F, et al. (2014) Endothelial microparticles as conveyors of information in atherosclerotic disease. Atherosclerosis 234: 295-302. [Crossref]

6. Dignat-Gorge F, Sampol J (2000) Circulating endothelial cells in vascular disorders new insights into and old concept. Eur J Haematol 65: 215-220. [Crossref]

7. Piccin A, Murphy WG, Smith OP 21 (2007) Circulating microparticles: pathophysiology and clinical implications. Blood Rev 21: 157-171. [Crossref]

8. Mahalle N, Kulkarni MV, Garg MK, Naik SS (2013) Vitamin B12 deficiency and hyperhomocysteinemia as correlates of cardiovascular risk factors in Indian subjects with coronary artery disease. J Cardiol 61: 289-294. [Crossref]

9. Ayer JG, Harmer JA, Nakhla S, Xuan W, Ng MK, et al. (2009) HDL-cholesterol, blood pressure, and asymmetric dimethylarginine are significantly associated with arterial wall thickness in children. Arterioscler Thromb Vasc Biol 296: 943-949. [Crossref]
10. McCully KS (1969) Vascular pathology of homocysteinemia: implications for the pathogenesis of arteriosclerosis. Am J Pathol 56: 111-128. [Crossref]

11. Valuck RJ, Ruscin JM (2004) H2 blocker or proton pump inhibitor use and risk of vitamin B12 deficiency in older adults. J Clin Epidemiol 45: 422-428. [Crossref]

12. Stabler SP (1995) Screening the older population for cobalamin (vitamin B12) deficiency. J Am Geriatr Soc 43: 1290-1297. [Crossref]

13. Helbing T, Olivier C, Bode C, Moser M, Diehl P (2014) Role of microparticles in endothelial dysfunction and arterial hypertension. World J Cardiol 65: 112-118. [Crossref]

14. Pawlak R (2015) Is vitamin B12 deficiency a risk factor for cardiovascular disease in vegetarians? Am J Prev Med 48: 11-26. [Crossref]

15. Harker LA, Ross R, Slichter SJ, Scott CR (1976) Homocysteine-induced arteriosclerosis: the role of endothelial cell injury and platelet response in its genesis. $J$ Clin Invest 58: 731-741. [Crossref]

16. Mudd SH, Levy HL, Skovby FD (1989) Disorders of transulfuration. In: Scriver C, Beaudet AL, Sly WS, The Metabolic Basis of Inherited Disease, 6th Edition, New York, 693-734.

17. McCully KS (1969) Vascular pathology of homocysteinemia: implications for the pathogenesis of arteriosclerosis. Am J Pathol 56: 111-128. [Crossref]

18. Pignoli P, Tremoli E, Poli A, Oreste P, Paoletti R (1986) Intimal plus medial thickness of the arterial wall: a direct measurement with ultrasound imaging. Circulation 74 1399-1406. [Crossref]

19. Libetta C, Villa G, Pirrelli S, Sepe V, Gori E, et al. (2001) Homocysteine plasma levels correlate with intimal carotid artery thickness in haemodialysis patients. Nephrol Dial Transplant 16: 2444-2445.

20. Bruyndonckx L, Hoymans VY, Frederix G, De Guchtenaere A, Franckx H, et al. (2014) Endothelial progenitor cells and endothelial microparticles are independent predictors of endothelial function. J Pediatr 165: 300-305. [Crossref]

21. Suhara T1, Fukuo K, Yasuda O, Tsubakimoto M, Takemura Y, et al. (2004) Homocysteine enhances endothelial apoptosis via upregulation of Fas-mediated pathways. Hypertension 43: 1208-1213. [Crossref]

22. Sekuła M, JanawaG, Stankiewicz E, Stępień E (2011) Endothelial microparticle formation in moderate concentrations of homocysteine and methionine in vitro. Cell Mol Biol Lett 16: 69-78. [Crossref]

Copyright: (2018 Dündar MA. This is an open-access article distributed under the terms of the Creative Commons Attribution License, which permits unrestricted use, distribution, and reproduction in any medium, provided the original author and source are credited. 